\title{
Pathophysiological mechanisms for the development of gestational diabetes insipidus
}

\author{
Rosa Diaz-Perez ${ }^{1 *}$ and Janet E Davis ${ }^{2}$ \\ ${ }^{1}$ MS3, University of Medicine and Health Sciences, 3219 Flowers Rd S, Apt G, Atlanta, Ga 30341, USA \\ ${ }^{2}$ Maternal Fetal Medicine, 1348 Walton Way, Suite 4300, Augusta, Ga 30901, USA
}

\section{Introduction}

Diabetes Insipidus is a rare complication that occurs in 2 to 4 per 100,000 pregnancies [1,2]. The diagnosis of gestational DI can be challenging because it usually presents with symptoms of polydipsia and polyuria, which are often attributed to normal pregnancy [3]. This condition usually develops between the second to third trimester of pregnancy and remits spontaneously 4 to 6 weeks after delivery [1]. The most common etiology for gestational DI is excessive vassopresinase activity leading to $\mathrm{ADH}$ degradation. The objective of this review is to consider other pathophysiological mechanisms for the development of Gestational DI, its clinical presentation and to understand different tests for diagnosis.

\section{Normal physiology during pregnancy}

Antidiuretic hormone $(\mathrm{ADH})$ is a peptide hormone that is synthesized in the supraopitic nucleus in the hypothalamus and released by the posterior pituitary. It acts on $\mathrm{V}_{2}$ renal receptors to regulate reuptake of water and $\mathrm{V}_{1}$ receptors in vascular smooth muscle to induce vasoconstriction. Normally, an increase in plasma osmolality, a reduction in blood pressure or a decreased renal perfusion will serve as the feedback mechanism for the release of $\mathrm{ADH}$. In turn, this hormone will lead to the transposition of aquaporins into the apical membrane of the collecting duct cells causing a reabsorption of water and creating an osmotic balance [4].

In normal pregnancy, there is a decrease in systemic vascular resistance (SVR) and a hormone-induced vasodilation. Consequently, the release of $\mathrm{ADH}$ is increased leading to an increased water reabsorption in the renal collecting duct. This reabsorption creates a new of hyponatremic hypervolemic state with a normal decrease in plasma osmolality about $270 \mathrm{mosmol} / \mathrm{kg}$ and a reduced plasma sodium level of 4 to $5 \mathrm{meq} / \mathrm{L}[2,3]$.

\section{Pathophysiology of gestational diabetes insipidus}

The development of gestational DI can be classified into different categories ranging from a preexisting subclinical DI enhanced in pregnancy to a transitional state that spontaneously regress at postpartum state. GDI is divided in the following:

\section{Pregnancy exacerbating symptoms of pre-existing DI.}

In rare cases, a patient can have an undiagnosed latent DI where there is a deficiency in the $\mathrm{ADH}$ secreting ability. Prior to pregnancy, it is possible for the patient to remain asymptomatic, but once the patient becomes pregnant the vasopressinase activity surpass the circulating levels of ADH. The symptoms become more apparent close to the third trimester where there is an increased production of vasopressinase by placental trophoblasts and a marked reduction in the ADH reserves, making the system unable to maintain homeostasis [5]. Pre-existing DI should be suspected if the patient has a recurrence of gestational DI in the next pregnancy with no impairment in liver function.

Patient also can have a diagnosed DI and, once the system is faced with a new physiological challenge such as pregnancy, symptoms are aggravated. In a normal non-pregnant state, it is possible for a patient with central DI treated with desmopressin to improve their symptoms to a point where medical therapy is no longer required. Once these patients begin a pregnancy therapy must be resumed until postpartum period [5].

\section{Transient Gestational DI}

DI during pregnancy is mainly related to an excessive activity of vasopressinase, which increases $\mathrm{ADH}$ degradation and clearance. This enzyme is expressed by placental trophoblasts from the $7^{\text {th }}$ gestational week and increases 1000 - fold between the $7^{\text {th }}$ and the $40^{\text {th }}$ week [1]. The amount of vasopressin is directly proportional to the size of the placenta; therefore, increased $\mathrm{ADH}$ clearance and DI can be associated with multiple gestations [6]. There are also reported cases of transient Gestational DI as a result of an increased production of renal prostaglandins, in particular $\mathrm{PGE}_{2}$, which decreases renal sensitivity to $\mathrm{ADH}$.

Normally, liver serves to metabolize vasopressinase and inactivates it, which maintains a circulating level of ADH during the pregnancy. Therefore, there is a strong association between liver dysfunction and the development of gestational DI. It is suggested that there is a link between gestational DI and Pre-eclampsia toxemia (PET) and HELLP.

Authors suggest two potential mechanisms that act together to lower the circulating levels of $\mathrm{ADH}$ in a pregnant female. The first mechanism proposes that there is a hepatic dysfunction secondary to de development of PET/HELLP syndrome that impairs the metabolism of vasopressinase. Increase activity of vassopresinase in circulation will increase the $\mathrm{ADH}$ clearance. The second mechanism states that there is a development of reversible pituitary hypoperfusion secondary to the vasoconstriction seen in PET/HELLP syndrome. This leads to a

${ }^{\star}$ Correspondence to: Rosa Diaz-Perez, 3219 Flowers Rd S, Apt G, Atlanta, Ga 30341, USA, TEL: 787-240-8764; E-mail: Rosadiazperez@hotmail.com; Rdiaz@umhs-sk.net

Received: September 15, 2018; Accepted: September 22, 2018; Published: September 29, 2018 
decreased $\mathrm{ADH}$ release from the posterior pituitary and favoring the development of Gestational DI [2].

\section{Autoimmune hypophysitis}

In autoimmune hypophysitis the targeted auto-antigen is Rabphilin $3 \mathrm{~A}$, which is expressed in the $\mathrm{ADH}$ neurons in the supraoptic nucleus in the hypothalamus. There are rare reports of pregnant women developing Gestational DI as secondary to the development of antiRabphilin $3 \mathrm{~A}$ antibodies during the third trimester. In these clinical cases, liver function is preserved $[7,8]$. The autoimmune state remit spontaneously after delivery but it usually takes more than 6 months for the regression of the inflammatory state ${ }^{3}$. Therefore, continuous medical treatment is required [8].

\section{Postpartum DI}

DI can occur after delivery trough different mechanism. These cases tend to be rare and sometimes can lead to a permanent DI. In the case of placental abruption, there can be a sudden release of vasopressinase to the maternal blood stream causing transient DI.

Peripartum hemorrhagic shock or significant bleeding can cause Sheehan syndrome. This syndrome is characterized by an anterior pituitary gland insufficiency but is not uncommon for the necrosis to extend to the posterior lobe. Also, increase activity of estrogen during pregnancy can cause pituitary tumors to grow. This tumor is at risk of hemorrhaging after delivery causing pituitary apoplexy. In both cases development of permanent DI is possible [8].

\section{Clinical presentation and diagnosis}

Gestational DI is classically presented with polydipsia and dilute polyuria [6]. The index of suspicion should increase when the intake of water is more than $3 \mathrm{~L} /$ day and the urine output is more than $3 \mathrm{~L} /$ day [2].

Laboratory data will most likely show a marked decrease urine osmolality along with an increased serum osmolality and sodium concentration [1]. During normal pregnancy plasma osmolality is reduced to about $270 \mathrm{mosmol} / \mathrm{kg}$ and a reduced plasma sodium level of 4 to $5 \mathrm{meq} / \mathrm{L}$ because of the pregnancy related dilution [3]. Therefore, plasma osmolality comparable to that of a non-pregnant woman or more than $>285 \mathrm{mosmol} / \mathrm{kg}$ should increase index of suspicion. This data is usually accompanied by plasma sodium comparable to borderline high in a non-pregnant women and urine osmolality under $300 \mathrm{mosmol} / \mathrm{kg}$ [5]. Measurement of urine to plasma osmolality ratio $<2$ is characteristic of significant gestational DI.

To determine that the $\mathrm{ADH}$ is low because of centrally lack of production, or as a product of degradation $\mathrm{ADH}$ can be assayed with the vasopressinase. Measurement of vasopressinase can also be done with chromatography.

In the case of autoimmune hypophysitis a titer for specific antibodies such as anti-rabphilin-3A can aid in the diagnosis. A follow-up cerebral MRI with contrast is helpful in evaluating hypothalamic-pituitary axis function after delivery.

Presence of glucose in the urine can be used to differentiate polyuria caused by uncontrolled DM from that in gestational DI. A water deprivation test can be useful to exclude a diagnosis of psychogenic DI. This test should be carried out with precautions and under close monitoring to avoid uteroplacental insufficiency secondary to severe dehydration [2]. Cerebral MRI can be useful to detect tumors. Close monitoring of urine osmolality after medical treatment can be helpful in making a differential between vasopressin- responsive and vasopressin resistant form of DI [5]. Pituitary function test to evaluate thyroid function, cortisol levels and prolactin levels should be performed if panhypopituitarism from Sheehan's syndrome or pituitary apoplexy is suspected [2].

\section{Maternal- Fetal consequences}

Reduced intravascular volume in women with Gestational DI can cause ineffectiveness of spinal anesthesia in case of the requirement of operative delivery. Alternative use of epidural anesthesia to establish surgical block should be considered. Women with DI should be referred to antenatal anesthetic review since the transmembrane sodium gradient rises and it can decrease the potency of local anesthetics [2].

Patients with PET and a co-existing gestational DI can have an adverse effect on prophylactic treatment for eclamptic seizures. Glomerular filtration rate (GFR) is increased during DI, in turn; it raises the renal excretion of magnesium sulfate. As a result of the change in diuresis PET patient can have a subtherapeutic magnesium level. Therefore, patient can benefit an increased magnesium infusion rate and close monitoring of levels.

The diagnosis and medical management of gestational DI lower the morbidity and mortality. Untreated DI can have a significant adverse effect in the mother and the fetus [5]. Severe dehydration and maternal acidosis can cause uteroplacental insufficiency causing fetal death. Some authors suggest that vasoprassinase can cross the placenta causing polyhydramnios and hypernatremia of the newborn [2]. Other cases of transient hypernatremia of the newborn have been reported with the maternal use of drugs that can be transferred to fetus trough placenta such as haloperidol. In such cases severe fetal dehydration and electrolyte imbalance can increase morbidity and mortality in newborns [9].

\section{Treatment}

The treatment of choice for Gestational DI is intranasal desmopressin [5]. 1-deamino-8-D- arginine (DDAVP or desmopressin) is an $\mathrm{ADH}$ analogue. Vasopressinase degrades $\mathrm{ADH}$ by withdrawing the amino acids from the $\mathrm{N}$ - terminal of this peptide; since desmopressin has a different $\mathrm{N}$-terminal it renders immunity to degradation by vasopressinase [2].

The goal of this medical therapy is to correct the osmotic imbalance. It is important to constantly monitor the sodium correction since it has to be a slow correction to avoid secondary brain swelling and neuronal damage. Sodium levels should be corrected at a rate of $1 \mathrm{mmol} /$ hour and the goal is to achieve a diuresis of no more than 2 to 3 liters per day [2]

Exposure to desmopressin during gestation does not increase chances of fetal malformations. It is safe to use during the entire pregnancy and during breastfeeding. DDVAP is secreted in the mother's milk in small quantities but it is poorly absorbed by the child's digestive tract, it is no known to cause any fluid or electrolyte imbalance in the baby. During post-partum the dose of DDVAP can be lowered as a consequence of increase stimulation of $\mathrm{ADH}$ secretion by breastfeeding [5].

\section{Discussion}

Gestational DI is a rare complication of pregnancy that usually begins between second to third trimester. There are different etiologies of DI but the most common is excessive vasopressinase activity. Placental trophobalst produce vasopressinase and its level are increased during pregnancy [5]. The liver normally metabolizes this enzyme; in 
such cases where there is an impairment of liver function the levels of vasopressinase accumulate causing a degradation of $\mathrm{ADH}$ that exceeds its secretion by the posterior pituitary. In turn, this causes an osmotic imbalance creating a hypernatremic hypovolemic state in the mother.

Untreated DI can lead to severe dehydration and uteroplacental deficiency increasing morbidity and mortality of the mother and fetus. It is important to raise levels of suspicion when patient reports excessive thirst and urination. Laboratory data, such as serum osmolality, urine osmolality and sodium levels can aid in the diagnosis of gestational DI. Once diagnosed, the treatment of choice is intranasal desmopressin, an $\mathrm{ADH}$ analogue. Medical therapy with desmopressin helps to create osmotic balance. Desmopressin is safe during pregnancy and lactation and does not cause an electrolyte in balance on lactating neonate. The effects of vasopressinase often remit spontaneously around a month after delivery and medical therapy is no longer indicated.

\section{References}

1. Marques P, Gunawardana K, Grossman A (2015) Transient diabetes insipidus in pregnancy. Endocrinology, Diabetes \& Metabolism Case Reports.
2. Quigley J, Shelton C, Issa B, Sripada S (2018) Diabetes insipidus in pregnancy [Abstract]. The Obstetrician \& Gynaecologist 20: 41-48.

3. Sum M, Fleischer JB, Khandji AG, Wardlaw SL (2017) Pitfall in the Diagnosis of Diabetes Insipidus and Pregnancy. Case Rep Obstet Gynecol 2017: 7879038. [Crosssref]

4. Knepper MA, Kwon TH, Nielsen S (2015) Molecular physiology of water balance. $N$ Engl J Med 372: 1349-1358. [Crosssref]

5. Aleksandrov N, Audibert F, Bedard MJ, Mahone M, Goffinet F, et al. (2010) Gestational Diabetes Insipidus: A Review of an Underdiagnosed Condition. $J$ Obstet Gynaecol Can., 32: 225-231. [Crosssref]

6. Razavi A (2017) Gestational Diabetes Insipidus (Gdi) Associated with Pre-Eclampsia [Abstract]. Womens Health 5(6).

7. Sakurai K, Yamashita R, Niituma S, Iwama S, Sugimura Y, et al. (2017). Usefulness of anti-rabphilin-3A antibodies for diagnosing central diabetes insipidus in the third trimester of pregnancy [Abstract]. Endocrine J 64: 645-650. [Crosssref]

8. Akar M, Kasapkara ÇS, Özbek MN, Tüzün H, Aldudak B, et al. (2014). Transient nephrogenic diabetes insipidus caused by fetal exposure to haloperidol. Renal Failure 36: 951-952. [Crosssref]

9. Bellastella G, Bizzarro A, Aitella E, Barrasso M, Cozzolino D, et al. (2014). Pregnancy may favour the development of severe autoimmune central diabetes insipidus in women with vasopressin cell antibodies: Description of two cases. Eur J Endocrinol 172: K11-7. [Crosssref]

Copyright: (C2018 Diaz-Perez R. This is an open-access article distributed under the terms of the Creative Commons Attribution License, which permits unrestricted use, distribution, and reproduction in any medium, provided the original author and source are credited. 